\title{
A Transmission Scheme for Streaming Variable Bit Rate Video over Internet
}

\author{
Sunghoon Son \\ Electronics and Telecommunications Research Institute, \\ 305-350 Taejon, Korea \\ sonsh@etri.re.kr
}

\begin{abstract}
In this paper, we consider transmission of variable bit rate (VBR) stored video for distributed streaming service. In streaming service over Internet, video server usually employs network congestion control protocol in order to adapt the dynamic change of network traffic. This protocol often results in the discontiguity of playback at client's side. We propose a novel transmission scheme to overcome this problem. Under the proposed scheme, video server prepares basic transmission schedule of video by off-line processing of VBR video. Using this information, server can control the amount of data transmitted, which enables client to avoid the discontinuity in playback, even if the allocation of network bandwidth changes during service.
\end{abstract}

\section{Introduction}

Recent advances in hardware technologies have made possible many distributed continuous media applications. Video server is one of the most essential component in these applications. A video server stores a large amount of video data on a large array of high-capacity storage devices. On receiving a playback request from a remote client, the video server services the client by retrieving the requested video data and then transmitting it to the client's site.

Video server must carefully transmit video data due to the real-time requirement of video playback. Network bandwidth used in streaming is one of the most important resources that video server manage. Since video streams are usually encoded using variable bit rate (VBR) encoding scheme such as MPEG standard [1, the amount of data needed in unit time for playback varies in time. This makes the problem much more complicated.

The usage of network bandwidth is typically affected by the transport protocol that video server uses. A distinguishing feature of media streaming protocols is its feedback control mechanism between server and client to dynamically adjust transmission rate according to network traffic [2], [3]. Under the current Internet environment, an open standard such as Real-time Transport Protocol (RTP) is commonly used protocol for the real-time transmission of video data. RTP is an IP-based protocol providing support for the transport of real-time data such as video [4]. RTP is designed to work in conjunction with the auxiliary control protocol Real-time Transport Control Protocol (RTCP) to get feedback on the 
quality of data transmission and information from participants in the sessions. Using RTP/RTCP, video server can change transmission rate dynamically based on the quality feedback from clients. Therefore a transmission scheme employed by video server should be aware of the operation of RTP/RTCP and dynamic QoS control mechanism based on the protocol.

In this paper, we consider the problem of continuous transmission of VBR video with the possible change of transmission rate during service. Especially we propose a new transmission scheme of VBR video considering feedback control mechanism for streaming over Internet. The proposed scheme first generates a continuous transmission schedule for a given video using fixed amount of network bandwidth reservation, which smoothes the bursty VBR video to reduce the rate variability. Then, another transmission technique is applied to the smoothed transmission schedule to adapt to the change of bandwidth allocation. All the procedures can be processed off-line. Some experimental results are given to demonstrate the performance of the proposed scheme.

The rest of this paper is organized as follows. In section 2, we introduce the notion of the continuous transmission and provide the review of the various VBR video transmission schemes. In section 3, we propose a new transmission scheme and its admission control algorithm that can adapt to the adjustment of transmission rate. Performance study is carried out in section 4 . Conclusion is given in section 5 .

\section{Continuous Transmission for VBR Video}

A video server transmits video frames periodically in round due to the periodic nature of video data [5]. The operation of client, the playback of video, is also periodic. A predefined number of video frames should be available at client's buffer during each round in order to guarantee the continuous playback. A video server often employs transmission scheduler to determine when and how much data should be sent to the client. Given a fixed amount of network bandwidth, a video server can accommodate only limited number of video streams concurrently. Before admitting a new client, a transmission scheduler must use an admission control procedure to verify whether there is enough network bandwidth available for the new client.

In case of stored VBR video, a priori information, such as a sequence of frame sizes, may be utilized to transmit VBR video frames efficiently. Using this information, the transmission scheduler can generate more efficient transmission schedule. Given a VBR video, transmission scheduler must determine the amount of video data to be transmitted during each round with no buffer starvation or buffer overflow at client site. A transmission scheduler must meet the following constraints for continuous transmission schedule it generates (See Table 1 for the definition of these quantities):

$$
\begin{gathered}
C(t) \leq P(t), 0 \leq t \leq n \\
P(t)-C(t) \leq B_{\max }, 0 \leq t \leq n
\end{gathered}
$$


Table 1. Notations

\begin{tabular}{ll}
\hline Notation & Definition \\
\hline$n$ & The length of video in time \\
$t$ & Time index \\
$t_{d}$ & Initial playback startup delay \\
$c(t)$ & Frame size at $t$, i.e. the amount of data consumed (displayed) \\
& by client at $t$ \\
$C(t)$ & The cumulative amount of data consumed by client \\
& during $[0, t], C(t)=\sum_{i=1}^{t} c(i)$ \\
$p(t)$ & The amount of data transmitted by server at $t$ \\
$P(t)$ & The cumulative amount of data transmitted by server \\
& during $[0, t], P(t)=\sum_{i=1}^{t} p(i)$ \\
$b(t)$ & Client's buffer level at $t$
\end{tabular}

$$
P(t+1)-P(t) \leq r, 0 \leq t \leq(n-1)
$$

(11) is the continuous transmission contraint, which means that the server always transmits more data than the client consumes for lossless and starvationfree playback. (2) is the client's buffer contraint, which means that the server transmits carefully not to overflow the limited client's buffer. And, (3) is the network bandwidth contraint, which means that the fixed amount of network bandwidth is allocated to each stream. Fig. 1 depicts various continuous transmission schedules that meet the above three constraints. Any non-decreasing lines between VBR trace (the thick solid line) and client's buffer level (the thick dotted line) can become continuous transmission schedules. 1

A lot of schemes have been proposed for the continuous transmission of VBR video. They mainly focused on the utilization of network bandwidth, minimization of rate variability, reducing buffer requirement, and so on. 6] demonstrates the fundamental limits and tradeoffs of providing a deterministic guarantee to VBR video traffic of live video traffic, which does not consider the stored property. In [7], an optimal smoothing scheme was proposed. The scheme is optimal in that it minimizes the client's buffer size and the rate variability of the VBR data. The authors tried to obtain statistical multiplexing gain too, but the statistical multiplexing gain from the optimally smoothed traffic is the lower bound of statistical multiplexing gain of the original traffic. The scheme proposed in [ 8 ] employs Constant Bit Rate (CBR) transmission of VBR data. Since the required bandwidth is fixed, multiplexing and admission control are very simple, which results in the reduced server load and no cell loss due to overload. However, this scheme tends to transmit a large amount of data unnecessarily because it always

${ }^{1}$ Although the operation of a video server is based on discrete time cycle, we will hereafter use continuous time notation to clearly convey the idea 


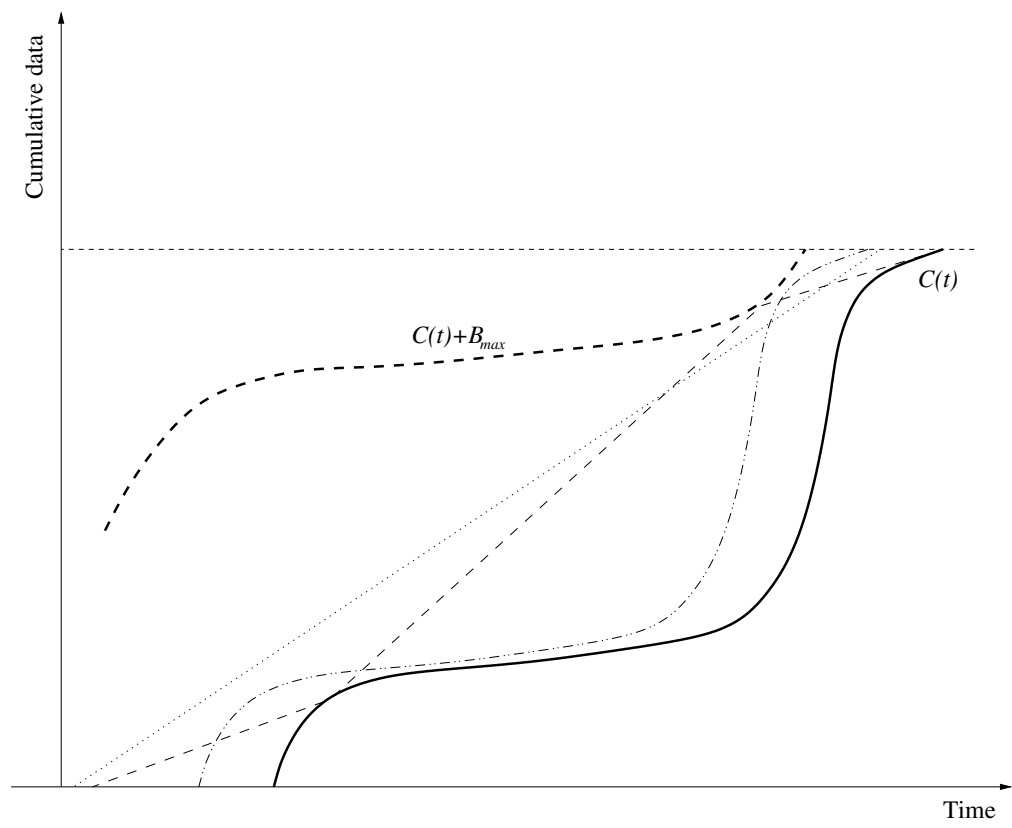

Fig. 1. Continuous transmission of VBR video and various transmission schedules

uses the entire bandwidth allocated to a stream, resulting in a large client buffer required.

\section{Continuous Transmission under Bandwidth Adjustment of CBR Channel}

In this section, we consider the continuous transmission of VBR video with the possible change of transmission rate during service. The scheme first generates a continuous transmission schedule for a given video trace based on the fixed amount of network bandwidth reservation, which smoothes the bursty VBR video to reduce the rate variability. Next, another transmission technique is applied to the transmission schedule to adapt to the change of bandwidth allocation. All the procedures described in this section can be processed off-line.

We assume that the video server uses CBR channel to transmit VBR video. We further assume that, when network traffic congestion occurs, the network bandwidth allocation of each stream may change during transmission due to the bandwidth renegotiation between client and server. We also assume that the client have limited buffer space which is usually less than a few mega bytes. 


\subsection{The Basic Transmission Schedule}

In this section, we introduce a transmission technique using CBR channel, which forms the basis of the transmission scheme considering bandwidth adjustment. The main idea is to transmit some large frames in advance when small frames are transmitted. This inevitably requires extra buffer space at client site.

In order to minimize the required buffer space at client for a given transmission rate, scan a VBR video's sequence of bit-rate backward from the end. Given a video trace, $c(t)$, and network bandwidth, $r$, the client's buffer level, $b(t)$, at each frame time can be calcaulated by

$$
\begin{aligned}
b(n) & =0 \\
\delta(t) & =c(t)-r+b(t+1), t=(n-1),(n-2), \ldots, 1 \\
b(t) & = \begin{cases}\delta(t), & \text { if } \delta(t) \geq 0 \\
0, & \text { otherwise }\end{cases}
\end{aligned}
$$

By starting at the last of the video frame, calculating backward using (4), we can increase the client's buffer level by the exact amount needed.

Fig. 2 illustrates an example of the basic transmission schedule obtained by the above procedure (the thick line in the figure). The intuition behind the figure can be explained as follows. Given a video's cumulative curve, $C(t)$, assume a straight line $L$ with the slope $r$. As we move $L$ right to left along the x-axis (time axis), $L$ and $C(t)$ can adjoin at several points. For example, in the figure, $L$ and $C(t)$ adjoin at $t_{3}$ and $L$ intersects $C(t)$ at $t_{2}$ at the same time. In this case, the transmission during the interval $\left[t_{2}, t_{3}\right]$ should be at the rate $r$. The reason is that there exists a point $t$ in $\left[t_{2}, t_{3}\right]$ such that the (average) bit rate in $\left[t, t_{3}\right]$ is higher than the bandwidth $r$. To avoid the discontinuity exptected in $\left[t, t_{3}\right]$, some video frames should be transmitted in advance during the interval $\left[t_{2}, t\right]$. As $L$ moves, we can identify such intervals easily. For the other type of intervals, like $\left[t_{1}, t_{2}\right]$, the transmission should be at the video frame's rate that is naturally less than the bandwidth allocation $r$.

The above procedure divides the whole transmission interval into two types of intervals. The one is the variable-rate transmission interval, during which transmission should be at the video frame's original rate (the interval (a) in Fig. 2). The video frame's rates in this interval is always smaller than the reserved transmission bandwidth $r$. The other is the fixed-rate transmission interval, during which transmission should be at the rate $r$ that is the fixed network bandwidth reserved (the interval (b) in Fig. 2). As a result, the whole transmission consists of alternating sequence of variable-rate intervals and fixed-rate intervals.

The client's buffer level at time $t$ is $b(t)=P(t)-C(t)$, and the client's maximum buffer requirement is given by $B_{\max }=M A X\{b(t)\}$. Therefore, the worst case startup delay is

$$
t_{d}=\frac{M A X\{b(t)\}}{r}, 0 \leq t \leq n
$$

Under the basic transmission, there exists a certain relation between transmission rate and client's buffer size. This is a unique characteristic of a particular 


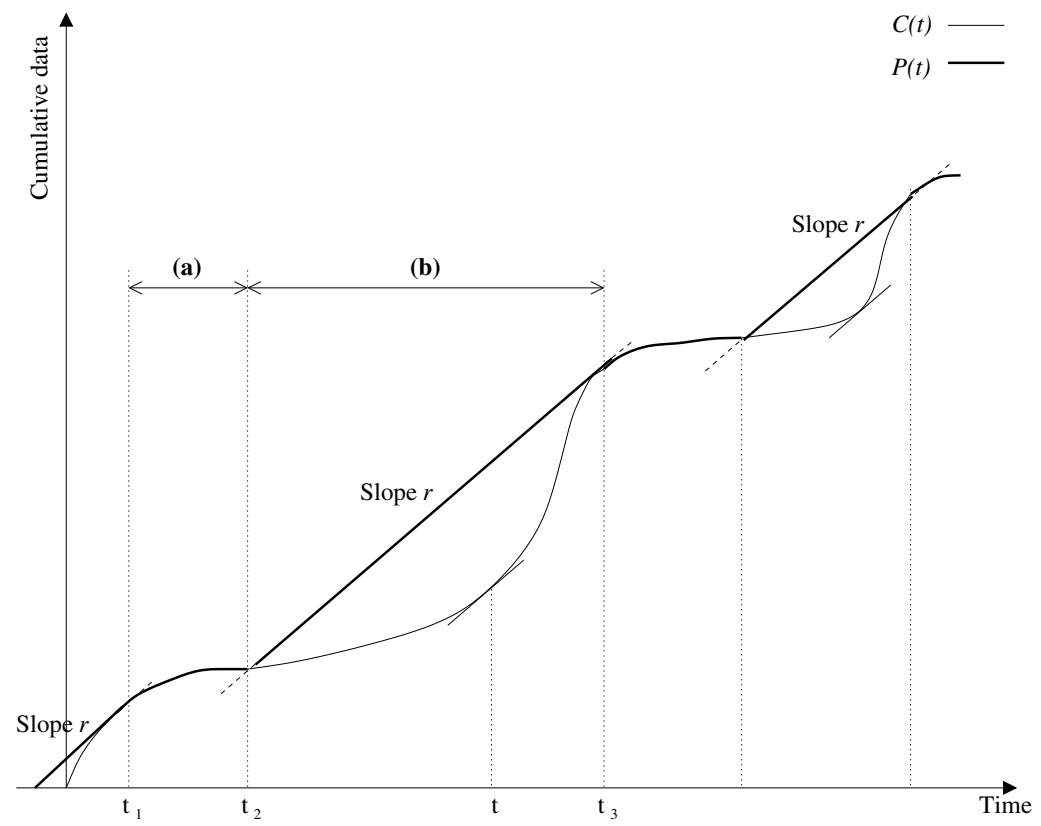

Fig. 2. Basic transmission schedule

video. Fig. 3 depicts a curve of real video's trace (which is used again for performace evaluation in Section 4) This curve is a useful meta data about VBR video, which can be utilized by video server for admission control of service requests. It can be utilized for the initial allocation of transmission rate too.

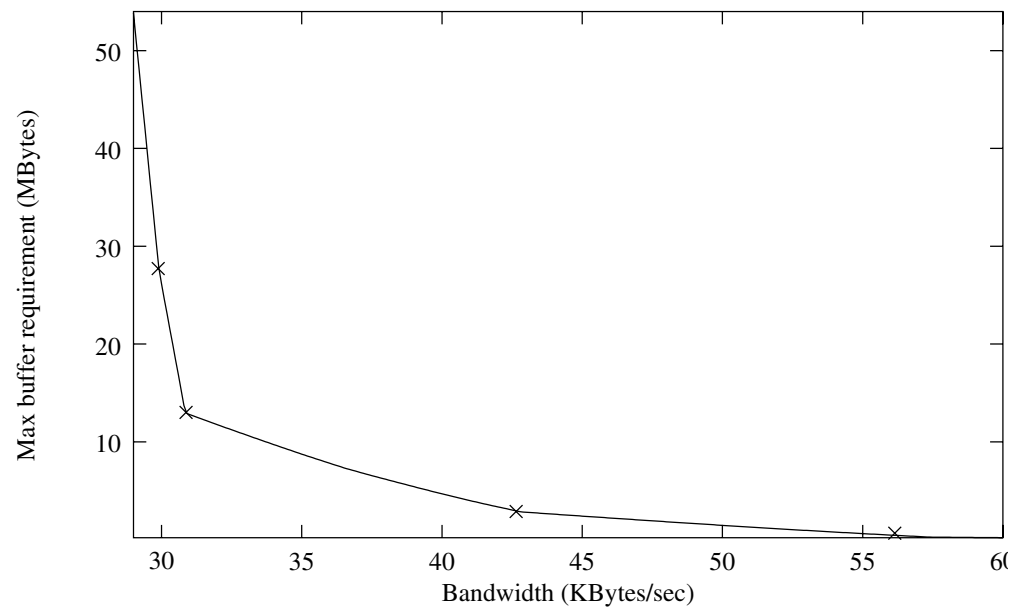

Fig. 3. Transmission rate vs. maximum client's buffer requirement 


\subsection{Continuous Transmission under Rate Adjustment}

Due to dynamic change of network traffic, media streaming over Internet often requires a congestion control mechanism. This significantly affects the determination of the bandwidth allocation and the calculation of transmission schedule. Here, we assume that a video server uses RTP/RTCP protocol as underlying congestion control mechanism.

RTP/RTCP typically operates as follows. A video server packetizes video into a series of RTP packets and sends it to client with timestamp and sequence number attached. Then, the client periodically sends RTCP packets, called receiver report, back to the server. Each receiver report contains reception quality feedback, including the highest packets number received, the number of packets lost, inter-arrival jitter, and timestamp to calculate the round-trip delay. Using an RTCP receiver report packet, the video server can estimate the current network state seen by the client and adjust transmission rate based on the estimation. An example of the mechanism can be found in [12. In our scheme, we only consider the case of decrease in transmission rate. The case of increase, which is very rare in real world, is trivial and not addressed here. We also assume that there exists a lower bound on the change of transmission rate.

Fig. 4 shows a transmission schedule of a stream with initial transmission rate is $r$. Consider a fixed-rate transmission interval beginning at $t_{r}$. Assume that a network congestion occurs at $t_{\text {reneg }}$, and the transmission rate decreases to $r^{\prime}\left(r^{\prime}<r\right)$ after congestion control. As shown by the figure, this will result in the discontinuity of playback at $t_{\text {discont }}$. The reason of the discontinuity is that the beginning of transmission with rate $r$ at $t_{r}$ is too late for continuous playback in case of the bandwidth decrease, although $t_{r}$ is the latest point at which the continuous transmission is possible with the minimum buffer requirement. This holds for any transmission start time between $\left[t_{r^{\prime}}, t_{r}\right]$, which is indicated by the shaded area and a thick dashed line in the figure. As shown by Fig. 4, to avoid the discontinuity in spite of bandwidth decrease can only be achieved by starting the transmission at $t_{r^{\prime}}$ or earlier. ( $t_{r^{\prime}}$ is the start time with the bandwidth $r^{\prime}$ ) However, this early transmission start time approach to avoid discontinuity may cause another problem. Assume that the transmission begins at time $t_{r^{\prime}}$ with the transmission rate $r$. This early transmission cause a buffer overflow at client side, which also results in the discontinuity of playback.

As a result, we have two constraints on transmission schedule, which should be met in order to guarantee continuous, overflow-free transmission under the bandwidth renegotiation. The one is that a transmission schedule should always be above the straight line with the slope $r^{\prime}$ (which is the minimum bandwidth exptected after the bandwidth renegotiation) in order to avoid discontinuity under the bandwidth renegotiation. The other is that there should be some brief pause (or decrease in the rate of transmission) so that the transmission schedule is always below the dashed line in order to avoid the buffer overflow at the client's side.

Fig. 5 depicts a possible transmission schedule that meets the both constraints. In the figure, the dashed line indicates client's maximum buffer require- 


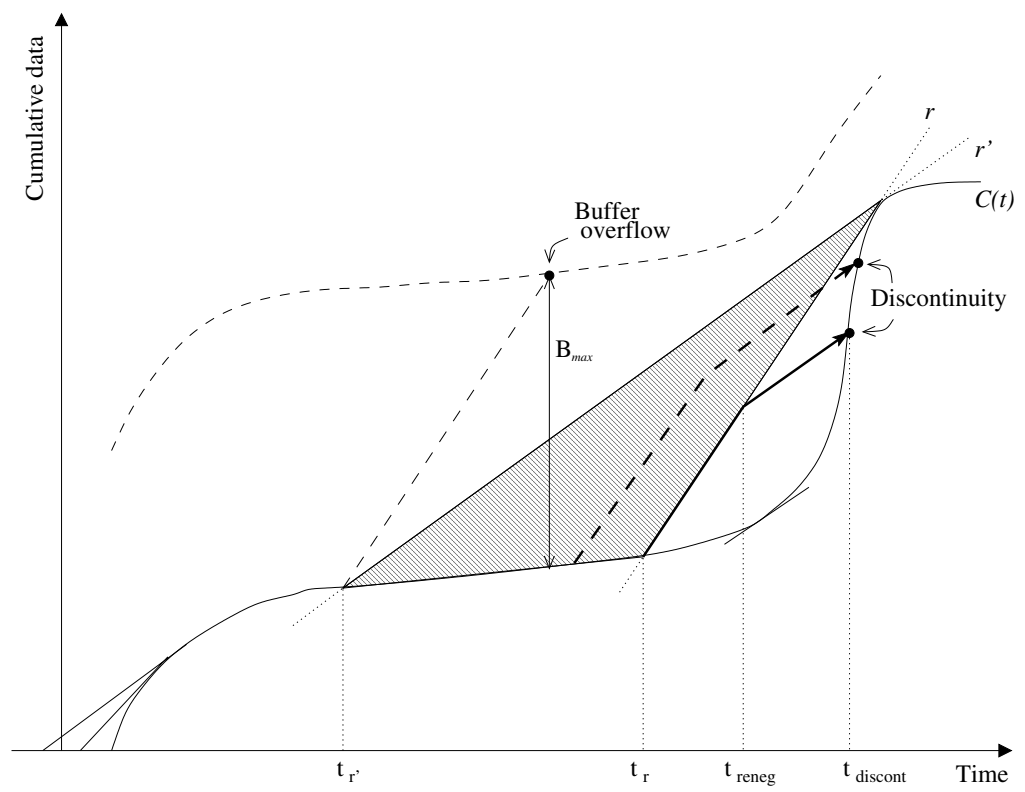

Fig. 4. Discontinuity due to the decrease in the bandwidth allocation

ments. Given two transmission rate $r$ and $r^{\prime}$ with $r^{\prime}<r$, the transmission scheme operates according to the basic transmission's intervals of whose bandwidth allocation is $r^{\prime}$, although it still utilizes the initial transmission rate $r$. The details of the operations are as follows. For variable-rate intervals of basic schedule, like $\left[t_{1}, t_{2}\right]$, server begins transmission at the rate of $r$. The transmission stops at $t_{p}$ where the cumulative amount of data transmitted becomes equal to the cumulative amount of video frames. The transmission pauses until the end of the variable-rate interval, $t_{2}$. For fixed-rate intervals, like $\left[t_{2}, t_{3}\right]$, server also begins at the rate of $r$, it stops at $t_{p^{\prime}}$ where client's buffer becomes full. It resumes at $t_{r}$ when the cumulative amount of data transmitted is equal to that of the basic transmission. It stops at $t_{p^{\prime \prime}}$ where the cumulative amount of data transmitted becomes equal to that of video frames at the end of the fixed-rate transmission interval, $t_{3}$. The thick line in the figure represents the newly suggested transmission schedule that can both avoid discontinuity and prevent overflow of client buffer.

\subsection{Calculation of Transmission Schedule}

The following is an algorithm to calculate the amount of data to be transmitted to client at each frame time under network bandwidth renegotiation. The algorithm first obtains the information about the intervals of the basic transmission schedule using the algorithm (line 1). This information includes type of interval (variable-rate interval or fixed rate interval), starting and end frames of interval 


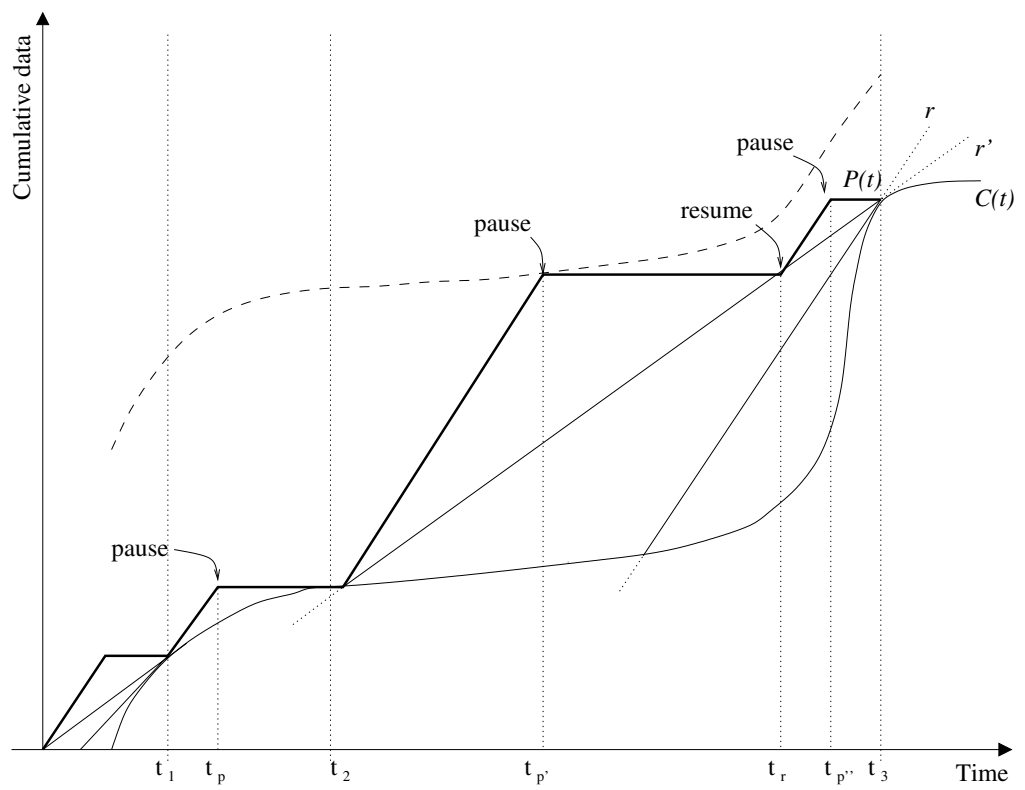

Fig. 5. New transmission schedule considering bandwidth renegotiation

$t_{s}$ and $t_{e}$, and the cumulative amount of video frames at these frame times $C\left(t_{s}\right)$ and $C\left(t_{e}\right)$. Then, the algorithm outputs $p(t)$ for each interval according to the method described in Fig. 5. The complexity of the algorithm is $O(m+n)$, where $n$ is the number of video frames and $m$ is the number of basic transmission schedule's intervals. This algorithm may be integrated with the transmission scheduler of video server. It can be utilized to off-line processing of video for more efficient transmission.

Transmitting a VBR stream considering rate adjustment

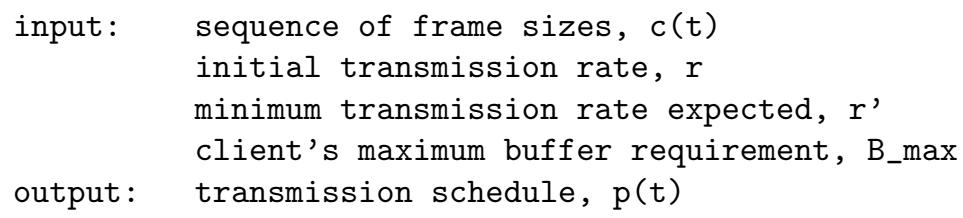

1 Identify fixed-rate \& variable-rate intervals using $r^{\prime}$;

2 for (each interval)

$3 \quad t_{-} s=$ starting frame time of interval;

$4 \quad t_{-} e=$ ending frame time of interval;

$5 \quad t=t_{-} s$;

6 if (variable-rate interval) then

$7 \quad$ p_sum $=\mathrm{C}\left(\mathrm{t}_{-} \mathrm{s}\right)$;

$8 \quad c_{-}$sum $=\mathrm{C}\left(\mathrm{t}_{-} \mathrm{s}\right)$; 


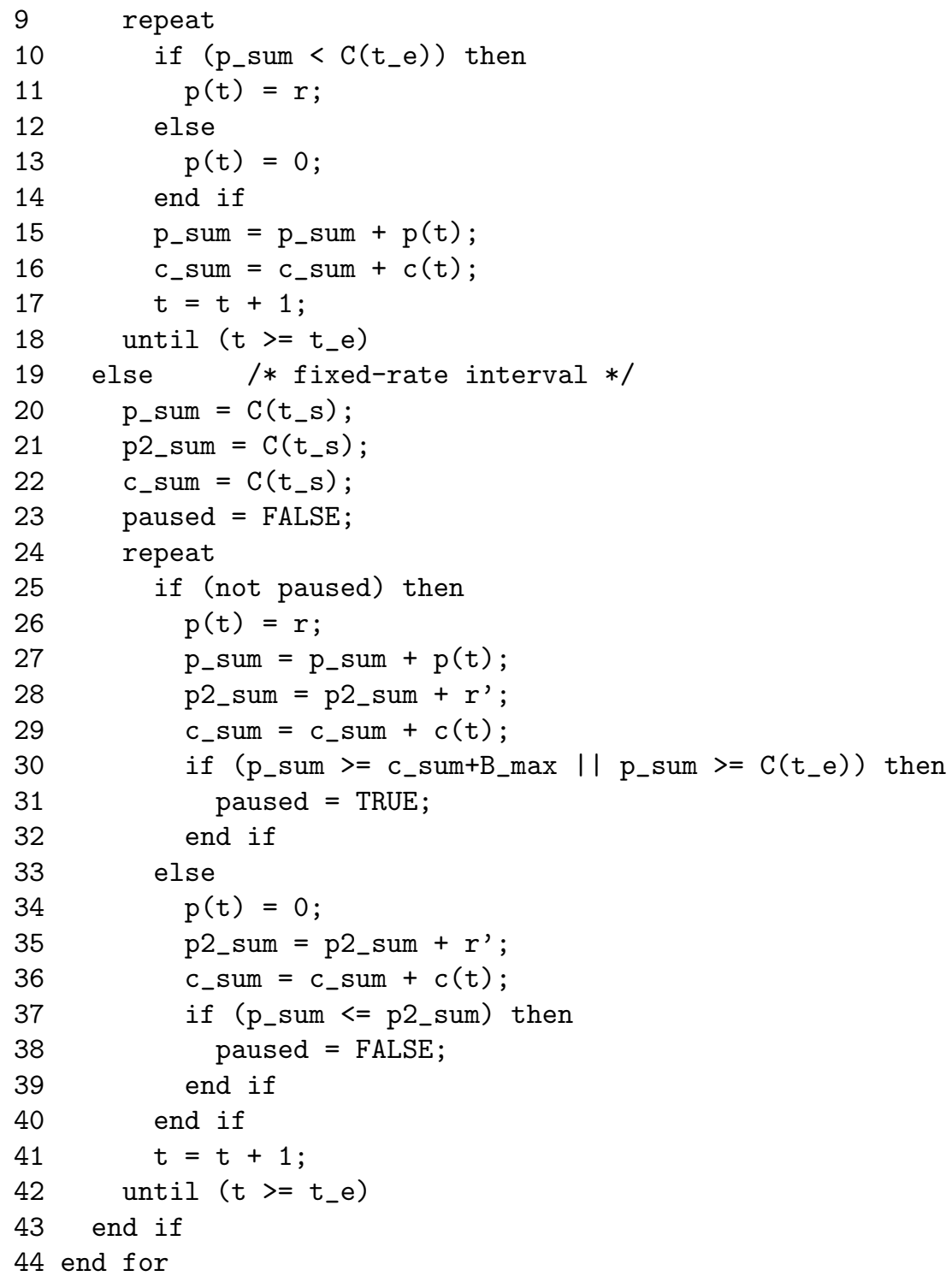

\section{Performance Analysis}

In this section, some experimental results are presented to evaluate the performance of the proposed transmission scheme. In theses experiments, we use the Starwars trace as our workload 2

Fig. 6] shows the distribution of variable-rate transmission intervals under the basic transmission scheme in section 3.1. As the reserved network bandwidth

${ }^{2} \mathrm{ftp}: / / \mathrm{ftp}$.bellcore.com:/pub/vbr.video.trace 
increases, the number of frame times belonging to the variable-rate intervals dramatically increases. It means that the most of frame times belongs to variablerate intervals during which the transmission is at the rate of video frame's rate. In other words, the basic transmission wastes most of network bandwidth, and the rate variability increases as the bandwidth allocation increases.

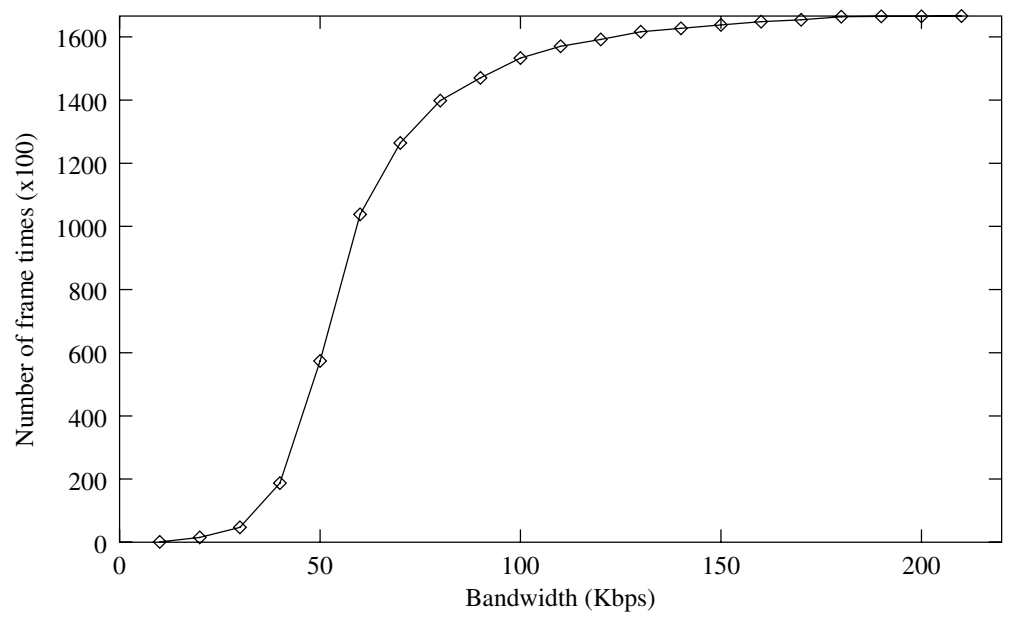

Fig. 6. The distribution of variable-rate transmission intervals

We compare the rate variability of the proposed transmission scheme with the optimal smoothing technique [7]. Table 2 shows the maximum/minimum transmission rates according to various client's buffer sizes. In our scheme, the transmission rate is constant except the case of brief pause in transmission (i.e. the minimum rate is 0 , which is omitted in the table). Our scheme seems to show slightly higher variability than the optimal one, but the difference between both schemes is closer as the client's buffer size increase. Moreover, the length of pausing intervals decrease as the client's buffer size increase, which reduces rate variability.

Table 2. Comparision of rate variablilty

\begin{tabular}{ccc}
\hline & optimal smoothing & proposed scheme \\
\hline Client buffer - 2 Mbytes & $43769 / 12938$ & 50033 \\
4Mbytes & $43769 / 19384$ & 48230 \\
8Mbytes & $43769 / 24743$ & 45928 \\
\hline
\end{tabular}

Fig. 7] compares the bandwidth utilization of the proposed transmission scheme with the basic one. As mentioned in section 3.2, in order to prevent the 
discontinuity of playback in spite of the decrease in bandwidth allocation, the scheme begin transmission much earlier than the basic scheme. And it have no variable-rate intervals. As a result, the scheme have better bandwidth utilization than the basic scheme.

On the contrary, the scheme have larger buffer requirement than the basic one (Fig. 8). The increase in the buffer usage is unavoiable, but the scheme guatantees that it does not exceed the maximum client's buffer space.

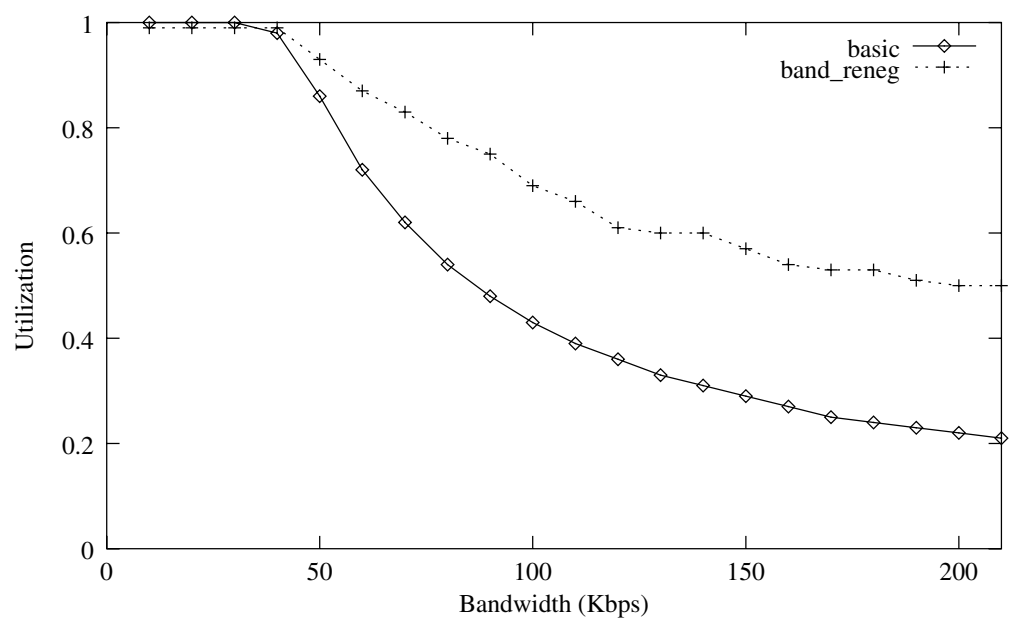

Fig. 7. The comparison of bandwidth utilization

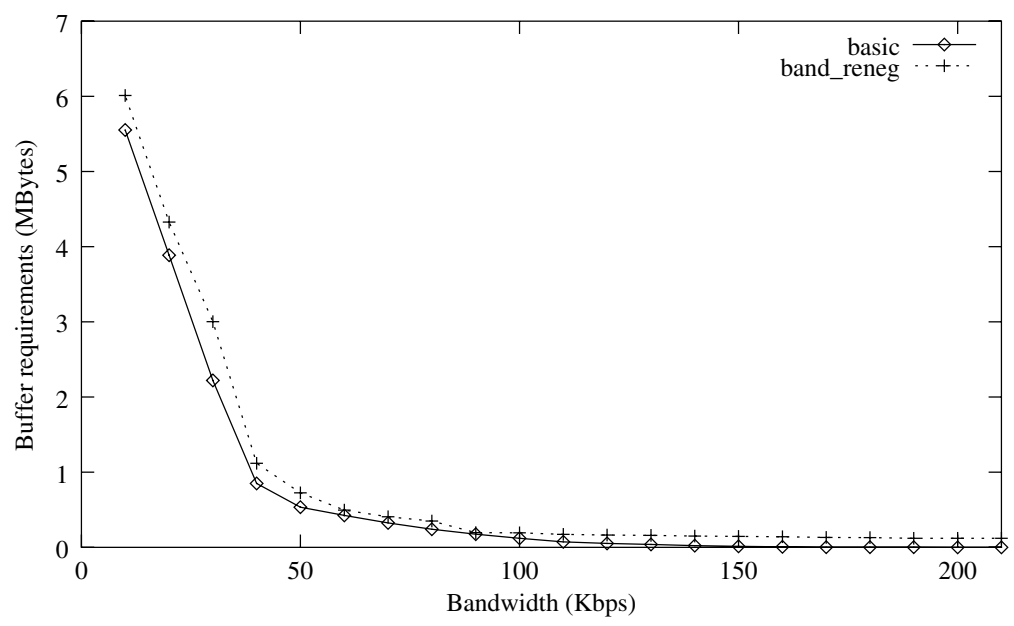

Fig. 8. The comparison of buffer requirement 


\section{Conclusion}

In this paper, we propose a new transmission scheme of VBR video considering feedback control mechanism for streaming over Internet. Given fixed-size client's buffer, the proposed scheme first calculates the basic transmission schedule. This can be done off-line using a priori information such as a sequence of frame sizes. Then, the scheme revises the transmission schedule in order to prepare for the rate adjustment during the transmission. Some experimental results are given to demonstrate the performance of the proposed scheme. The scheme shows higher rate variability than the optimal smoothing scheme

\section{References}

1. D. Le Gall, "MPEG: A video compression standard for multimedia applications", Communications of the ACM, Vol. 24, No. 4, pp. 59-63 1991.

2. T. T. J.-C. Bolt and I. Wakeman, "Scalable feedback control for multicast video distribution in the internet", In Proceedings of ACM SIGCOMM '94, pp. 58-67, September 1994.

3. H. Zhang and E. W. Knightly, "Red-vbr: a renegotiation-based approach to support delay-sensitive vbr video", ACM Multimedia Systems, vol. 5, pp. 164-176, May 1997.

4. H. Schulzrinne, S. Casner, R. Frederick, and V. Jacobson, "RTP: a transport protocol for real-time applications", RFC1889, 01/25/96.

5. D. J. Gemmell, H. M. Vin, D. D. Kandlur, P. V. Rangan, and L. A. Rowe, "Multimedia storage servers: a tutorial and survey", In IEEE Computer, Vol. 28, No. 5, pp. 40-49, May 1995.

6. E. W. Knightly, D. E. Wrege, J. Liebeherr, and H. Zhang, "Fundamental limits and tradeoffs of providing deterministic guarantees to vbr video traffic", In Proceedings of ACM SIGMETRICS '95, pp. 98-107, May 1995.

7. J. Salehi, Z.-L. Zhang, J. Kurose, and D. Towsley, "Supporting stored video: reducing rate variability and end-to-end resource requirements through optimal smoothing", In Proceedings of ACM SIGMETRICS '96, pp. 222-231, May 1996.

8. J. M. McManus and K. W. Ross, "Video on demand over atm: constant-rate transmission and transport", In Proceedings of IEEE INFOCOM '96, pp. 1335-1362, March 1996.

9. S. Paek and S. Chang, "Video server retrieval scheduling for variable bit rate scalable Video", In Proceedings of IEEE Int'l Conference on Multimedia Computing and Systems, pp. 108-112, June 1996.

10. N. Shroff and M. Schwartz, "Video modelling within networks using deterministic smoothing at the source", In Proceedings of IEEE INFOCOM '94, pp. 342-349, June 1994.

11. D. Ferrari and D. Verma, "A scheme for real-time channel establishment in widearea networks", IEEE Journal on Selected Area in Communications, Vol. 8, pp. 368-379, April 1990.

12. I. Busse, B. Deffner, and H. Schulzrinne, "Dynamic qos control of multimedia applications based on RTP", Computer Communications, Vol. 19, pp. 49-58, 1996. 